\title{
Nachbarschaften machen. Qualifizierung von Stadträumen zu Orten der interkulturellen Begegnung - Praxisbeispiele des lokalen Integrationsmanagements in Berlin
}

\author{
Dominik Haubrich
}

\section{$1 \quad$ Einleitung}

Seit den 1970er-Jahren hat sich ein interdisziplinäres und heterogenes Forschungsfeld rund um Stadtteile und Nachbarschaften, Kieze und Quartiere entwickelt. In aktuellen Debatten finden sich Definitions- und Abgrenzungsversuche, die sich über die Betonung individueller Zuschreibungsprozesse, raumbezogener Attribute, kollektiver Interaktionsprozesse, lokaler Netzwerke und Identitätsprozesse, alltäglicher Lebenswelten und sozialer Konstruktionen der Quartiers- und Nachbarschaftskategorie in Gestalt eines pragmatisch gelesenen Eklektizimus nähern (vgl. Schnur 2008, 2012). Sie verschneiden das Untersuchungsfeld von Quartieren und Nachbarschaften jeweils mit Konzepten wie community, sense of place, practices und emotions (vgl. Vogelpohl 2008; Deffner 2013). Mit der sukzessiven Integration sozialtheoretischer Ordnungskonzepte bei der Betrachtung von Stadtteilräumen treten zunehmend auch gesellschaftsrelevante Phänomene und ihre räumliche Bedeutung in den Vordergrund. Eine Abkehr von einem Zugang, der nicht zuvorderst seinen Blick auf den empirisch beobachtbaren Raum wirft, verlangt nach einer sozialwissenschaftlichen Perspektive auf räumliche Entwicklungen, die eine prozessorientierte Konzeptualisierung von Räumen denkt.

Der vorliegende Beitrag setzt sich mit der gesellschaftlichen Relevanz der hohen Zuwanderung von Geflüchteten nach 2015 auseinander. Indem er eine städtische Governance-Perspektive auf Integration um ordnungstheoretische Konzepte sozialer Praktiken erweitert, werden die sozialräumlichen Möglichkeiten und Implikationen der sich für lokale Ebenen genuin ableitenden Aufgaben der Integrations- und Begegnungsprozesse diskutiert. Im Einzelnen wenden sich die empirischen Überlegungen der lokalräumlichen Ebene der kommunalen Integrationspolitik zu. In aktuellen Projekten der Willkommenskultur, Akteurvernetzung und Stadtteilarbeit setzen Sozialunternehmungen ein solches prozessuales Raumverständnis praktisch um. Der Fokus der verschiedenen Projekte des lokalen Integrationsmanagements liegt dabei auf einem systematischen Aufbau von Kom- 
munikations- und Koordinationsstrukturen, welche die Aktivierung, die Begleitung und den Austausch von Neu- und Altberlinerinnen unterschiedlicher Herkunft niederschwellig ermöglichen und bestimmte Stadtteilräume zu Orten der interkulturellen Begegnung qualifizieren. In der Praxis stellen sich das Zusammenbringen und Begleiten von neuen und alten Stadtbewohner*innen als eine Vielzahl unterschiedlich organisierter und strukturierter Interaktionsprozesse auf lokaler Ebene dar. Unter Rückgriff auf das sozialräumliche Ordnungskonzept der Theorie sozialer Praktiken nach Schatzki (2002) argumentiert der Beitrag weiter, dass sich in der Verzahnung von beteiligten Akteur*innen und gemeinschaftlich erfahrenen Handlungszusammenhängen neue Ordnungsformationen von Nachbarschaften erkennen lassen. Durch die Wiedergabe erfahrungsbasierter Erkenntnisse in der Umsetzung aktueller Projekte des lokalen Integrationsmanagements werden schließlich Möglichkeitsräume aufgezeigt, in denen pragmatische Lösungswege für eine Gestaltung neuer Governance-Arenen der Integration aufgespannt werden.

\section{Wir schaffen das: die Verortung der Willkommenskultur in den Jahren 2015 und 2016}

In den Augusttagen 2015 spielten sich in Deutschland und Europa zahlreiche Geschehnisse ab, die das framework der Integrationspolitik zunächst, und u. U. nachhaltig ändern sollten. Am 19.08.2015 stellte der damalige Bundesinnenminister Thomas de Maizière seinen Bericht über die aktualisierte Prognose der Zuwanderung von Geflüchteten nach Deutschland vor. Er bekannte, dass im Jahr 2015 mit dem Zugang von 800.000 Geflüchteten zu rechnen sei, eine Zahl, die sich zwölf Monate später bestätigen sollte. Damit wurden Daten veröffentlicht, die das Doppelte der Frühjahrsprognose darstellten und die das Vierfache der Zuwanderung von Geflüchteten im Jahr 2014 beschrieben. De Maizière deutete auf die Herausforderung der Flüchtlingszuwanderung für Bund, Länder und Kommunen hin und betonte, dass man sich ihr gemeinsam annehmen müsse und sie gemeinsam meistern würde. Neue Wege seien zu gehen und pragmatische Lösungen zu finden (vgl. de Maizière 2015). Eine Woche später berichtete der damalige SPD-Parteivorsitzende Sigmar Gabriel über seine Erfahrung und die Arbeit der ehrenamtlichen Helfer*innen und der Behörden in der zu dieser Zeit größten Erstaufnahmeeinrichtung in Giessen (Hessen). Neben das Bekenntnis zu neuen Wegen und Lösungen gesellte sich das Lob der Kraft des Staates und die große Menschlichkeit. Mit dem gemeinsamen Streben von Akteur*innen könne Deutschland „einer großen Zahl von Menschen eine sichere Heimat geben“. Er sei sich sicher: „Wir schaffen das“" (Gabriel 2015).

Auf der Sommerpressekonferenz der Bundeskanzlerin am 31.08.2015 verkündigte Angela Merkel schließlich erstmals den Satz „Wir schaffen das“. Der 
Satz traf ins Rückenmark der Debatte, polarisierte Anhänger*innen wie Gegner*innen und war zugleich ganz bewusst gewählt:

„Ich sage ganz einfach: Deutschland ist ein starkes Land. Das Motiv, mit dem wir an diese Dinge herangehen, muss sein: Wir haben so vieles geschafft - wir schaffen das! Wir schaffen das, und dort, wo uns etwas im Wege steht, muss es überwunden werden, muss daran gearbeitet werden. Der Bund wird alles in seiner Macht Stehende tun zusammen mit den Ländern, zusammen mit den Kommunen -, um genau das durchzusetzen“ (Angela Merkel, Sommerpressekonferenz 31.08.2015).

Doch die Geschehnisse dieser Tage nahmen weiter Fahrt auf. Am 05.09.2015 beschloss Angela Merkel mit ihrem österreichischen Amtskollegen, den am Budapester Bahnhof festsitzenden Geflüchteten die Ausreise aus Ungarn und die Einreise nach Österreich und Deutschland zu ermöglichen. In der Folge dieser Entscheidung, die mit einer Aussetzung der Grenzkontrolle zwischen Deutschland und Österreich über mehr als eine Woche einherging, fanden immer mehr Menschen über die Balkanroute den Weg nach Deutschland. Die Maxime „Wir schaffen das" der Bundeskanzlerin wurde in den Folgejahren immer mehr zur Trennlinie zwischen den beiden regierenden Schwesterparteien CDU und CSU - verhandelt als Obergrenze mit seinem bekannten Vertreter Horst Seehofer -, aber auch zum Streitpunkt der Großen Koalition (2013-2017). So distanzierte sich etwa der SPD-Chef Gabriel ein Jahr später, insbesondere auch im Zusammenhang mit den ersten terroristischen Anschlägen in Würzburg am 18.07.2016, in München am 22.07.2016 und in Ansbach am 24.07.2016, von der einfachen Wiederholung von „Merkels Satz“, indem er betonte: „Der Satz klingt schön, aber er reicht nicht aus“ (zitiert nach Heißler 2016).

Die in den Jahren 2015 und 2016 formulierten Schlaglichter der bundesdeutschen Debatte um die Aufnahme, das Willkommenheißen und die Integration von Menschen mit aktueller Fluchtbiografie deuten auf einen zweiten Paradigmenwechsel der Integrationspolitik seit 2013 hin (vgl. Abb. 1). Davor hatten das Zuwanderungsgesetz von 2005 sowie die Etablierung von Integrationsgipfel und Nationalem Integrationsplan das Gerüst für die verwaltungspolitische Betonung der ressortübergreifenden Querschnittsaufgabe der Integration mit ihrem zentralen Instrument der gesamtgesellschaftlichen Debatte um Willkommenskultur geschaffen. Mit der Erweiterung der Integration von Zuwanderer*innen um die Gruppe der Geflüchteten und Ayslsuchenden wird seit 2013 schließlich der zweite Paradigmenwechsel der jüngeren Entwicklung der Integrationspolitik eingeläutet. Charakteristisch hierfür ist das verwaltungstechnische Verschneiden von Asylbzw. Flüchtlingspolitik und Arbeitsmarktpolitik seit Ende 2013. Das Verschneiden findet seitdem seinen Ausdruck in der Flexibilisierung des Arbeitsmarktzugangs bei gleichzeitiger Differenzierung der Zuwanderungsgruppen nach Her- 
kunftsländern. Arbeit, Sprache, Asylrecht und Sozialraum werden zu Schnittfeldern der kommunalen Integrationspolitik. Die Fokussierung der Arbeitsmarktintegration hat schließlich weitreichende Implikationen für das politische System. Integrationsmaßnahmen jenseits des Arbeitsmarkts unterliegen seitdem komplexeren vertikalen und horizontalen Kompetenzverteilungen entlang der Schnittstellen Gesetzgeber, Verwaltung, öffentliche Träger sowie privat- und zivilgesellschaftliche Akteur*innen. Die Gemengelage aus integrationsrelevanten Akteur*innen hat ihrerseits direkte Implikationen für die Gestaltung von Begegnungs- und Austauschformaten auf lokalräumlicher Ebene der Quartiere.

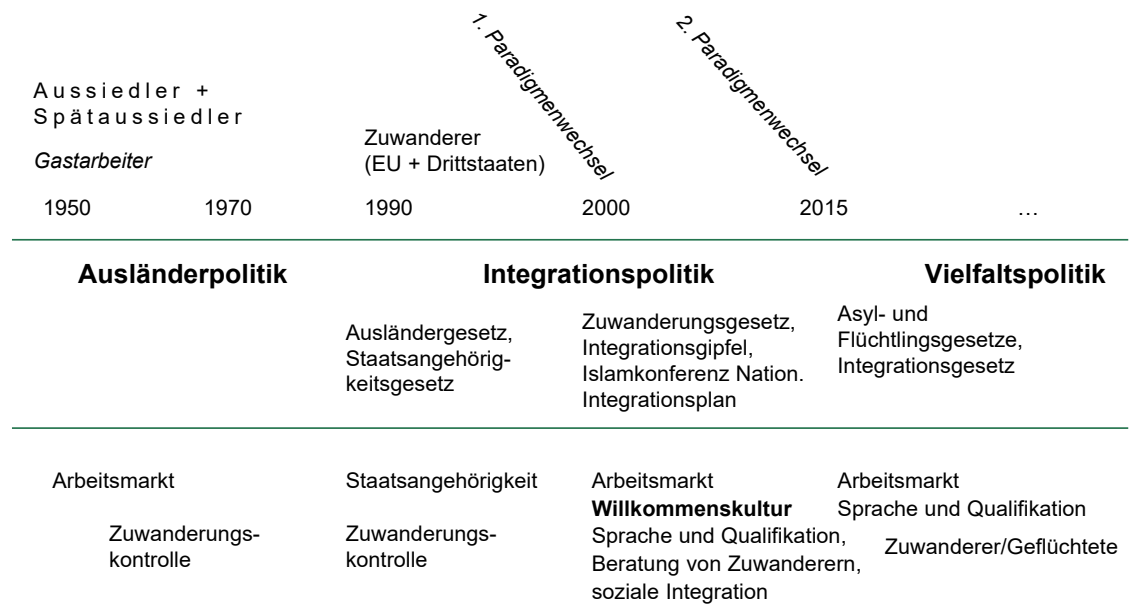

Abbildung 1: $\quad$ Paradigmenwandel in der Integrationspolitik

Quelle: eigene Darstellung

\section{3 „So schaffen wir das": das Wie der kommunalen Integrationspolitik}

Es gehört zwischenzeitlich zum fächerübergreifenden Konsens, dass der lokalen Ebene bei der sozialen Integration eine Schlüsselrolle zukommt (vgl. Saunders 2010). Städte, Stadtviertel und Quartiere sind die Motoren der Vielfalt (Bukow et al. 2011). In urbanen Stadtgesellschaften sind Migrant*innen in Arenen urbaner Ökonomien, Bildungs- und Arbeitssysteme sowie soziale Netzwerke eingebunden. Auf der lokalen Ebene kommt es zu Begegnung, Austausch und gesellschaftlicher Beteiligung. Im öffentlichen Begegnungsraum der Nachbarschaften und Wohnumfelder werden Anerkennung, soziale Kontrolle, Vertrauen und gesellschaftlicher Konflikt diskursiv und alltagspraktisch ausgehandelt. Dialog durch 
Vielfalt, Integrationserfolge und sozialräumliche Konflikte finden oftmals in direkter Nachbarschaft statt (vgl. Gesemann 2016). Im integrationspolitisch relevanten Werk „So schaffen wir das“ von Schiffauer et al. (2017) wird entlang von 90 Projekten der ehrenamtlichen und verwaltungspolitischen Arbeit mit Geflüchteten exemplarisch der Druck der raschen Unterbringung, Versorgung und sozialen Integration aufgezeigt, dem sich Kommunen im Zuge der Fluchtzuwanderung in den Jahren 2015 und 2016 noch einmal verstärkt gegenüber sahen. Aumüller und Gesemann (2014) weisen darauf hin, dass sich Kommunen deshalb schon seit Längerem - und damit weit vor der bundesdeutschen Ebene (entsprechend dem Diskurs im August 2015) - durch pragmatische Lösungen bei der Suche nach Integrationsmöglichkeiten auszeichneten. Um dem genuin lokalräumlichen Zielkonflikt aus Integration von Flüchtlingen und Migrationskontrolle gerecht zu werden, sind Kommunen insbesondere auf Nachbarschaftsebene auf ganzheitliche und kosteneffiziente Integrationslösungen angewiesen (vgl. Gesemann 2017). Infolge der kontinuierlichen Suche nach ganzheitlichen Wegen vereint sich im Spektrum integrationspolitischer, kommunaler Handlungsfelder (Sprachförderung, berufliche Integration, sozialräumliche Integration, politische Teilhabe und bürgerschaftliches Engagement) eine immer größere Zahl von Akteur*innen aus Verwaltung, Zivilgesellschaft und privatwirtschaftlichem bzw. gemeinnützigem Sektor. Aus der Governance-Perspektive kommunaler Integrationspolitik bildet die Bewältigung der Querschnittsaufgabe einerseits eine große Herausforderung. Andererseits schafft die Gemengelage aus Akteur*innwn und Praktiken im Zeichen der verwaltungstechnischen Krise des Zuzugs von Geflüchteten in kurzer Zeit auch einen produktiven Prozess (vgl. Schiffauer et al. 2017).

Um diesen produktiven Prozess der Entstehung von (neuen) Nachbarschaften zugänglich zu machen, bedarf es einer sozialtheoretischen Perspektivenerweiterung, die Raum als konstitutives Element des Sozialen versteht. Hierfür bietet sich die Theorie Sozialer Praktiken nach Schatzki (2002) an, bei der Raum sowohl konstitutive Voraussetzung als auch Ergebnis des Sozialen ist (vgl. Everts et al. 2011). Weder Raum noch das Soziale sind dabei präexistent bzw. stehen im Mittelpunkt der Betrachtung. In Erweiterung der wissenschaftlichen Debatte um die Kategorien von Quartieren und Nachbarschaften richtet die Praktikentheorie ihren Blick vielmehr auf das Tun und Sagen (Praktiken) sowie auf Materialitäten und Diskursformationen (Ordnungen) als die beiden zentralen Theoriebausteine (vgl. Abb. 2). Im Geflecht aus Praktiken und Ordnungen entsteht kontinuierlich der Aushandlungsort sozialer Ordnung (der sogenannte site), in dem und durch den soziales Zusammenleben stattfindet. Raum ist damit in wechselseitige Beziehungen von Praktiken und Ordnungen relational eingebunden: „Spaces [...] are preeminently qualified to be something where, and as part of which, events occur and entities exist" (Schatzki 2002: 140). Diese Perspektive lässt sich auf räumliche Phäno- 

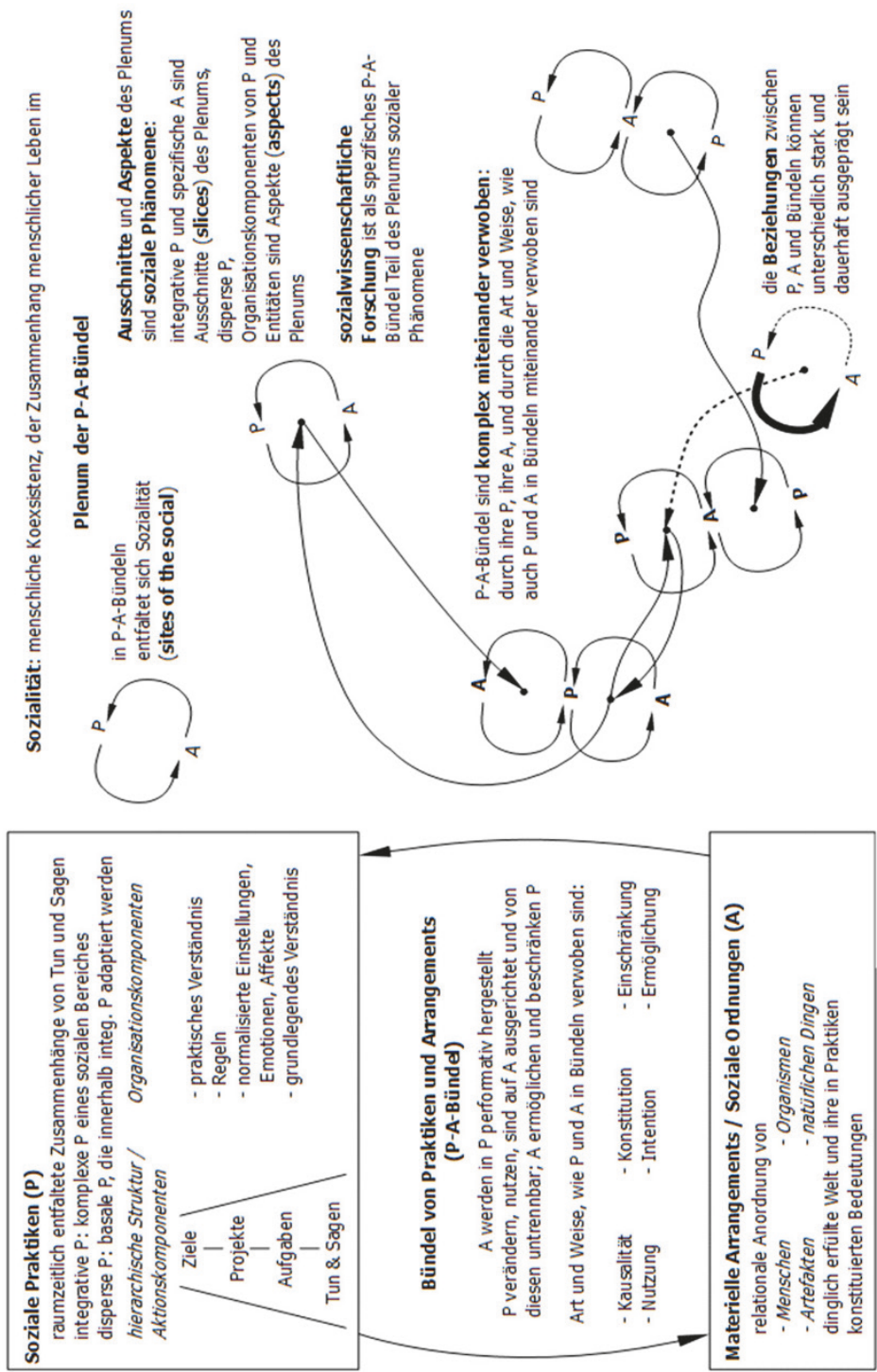

Abbildung 2: Konstitution sozialer Phänomene im praktikentheoretischen Vokabular Theodore Schatzkis

Quelle: Maus (2015: 59) 
mene und damit auf die Betrachtung des Hervorbringens und Machens von Nachbarschaften übertragen. Mit der sukzessiven Integration sozialtheoretischer Ordnungskonzepte bei der Betrachtung von Stadtteilräumen treten zunehmend gesellschaftsrelevante Phänomene und ihre raumrelevante Bedeutung in den Vordergrund. Am Beispiel der kommunalpolitischen Integrationsaufgabe soll im Anschluss ein Blick auf eine prozessorientierte Konzeptualisierung von Sozialräumen geworfen werden, in der die praktikentheoretischen Bausteine als Übersetzungsmethodik von diskursgeleiteten und anwendungsorientierten Praktiken der kommunalen Integrationspolitik dienen.

\section{Neue Nachbarschaften: das Beispiel Tempelhofer Feld}

Als lokalräumlichen Schnittpunkt der Kontextualisierung einer aktuellen Integrationspolitik diskutiert der vorliegende Beitrag das Berliner Beispiel des öffentlichen Freiraums Tempelhofer Feld. Nach einer kurzen stadtgeografischen Einordnung des Tempelhofer Feldes trägt der Beitrag die empirischen Notizen zweier Beispiele der Aushandlungen und Gestaltungen des lokalen Integrationsmanagements zusammen, die aus praktikentheoretischer Perspektive sodann als site der Entstehung neuer Nachbarschaften skizziert werden sollen.

Das Tempelhofer Feld, ein 2008 endgültig stillgelegter Flughafen, befindet sich in zentraler innerstädtischer Lage Berlins und weist mit einer Fläche von mehr als 328 Hektar ein großes Nutzungspozential auf. Seit der 1990er-Jahren ist das ehemalige Flughafengelände Bestandteil einer intensiven Debatte über Folgenutzung, Stadtrechtsfragen, Immobilieninteressen und Verwertungslogiken und neuerdings ist das Gelände eben auch der Aushandlungsort der kommunalen Integrationspolitik. Auf dem Flughafen Tempelhof konzentrieren sich daher auch Verhandlungsprozesse einer zivilgesellschaftlich organisierten Gruppe, die sich aus unterschiedlichen losen Bündelungen Mitte der 2000er-Jahre zur Initiative „100\% Tempelhofer Feld“ zusammengefunden hat. Ziel der Initiative war das Anstoßen und schließlich erfolgreiche Umsetzen eines Volksentscheids im Jahr 2014. Mit dem Volksentscheid wurde das „Gesetz zum Erhalt des Tempelhofer Feldes“ ins Berliner Abgeordnetenhaus getragen und Mitte 2014 erfolgreich verabschiedet (vgl. Senatsverwaltung für Justiz und Verbraucherschutz 2015).

Das „ThF-Gesetz“ regelt im Kern, dass die Bebauung des Tempelhofer Feldes vollkommen untersagt ist und bis auf Ausnahmen keine Wohn- oder Freizeitbebauung stattfinden kann. Mit der Gesetzeseinführung wurde zudem ein aufwendiges partizipatives Planungsinstrument definiert, dass die zukünftige Nutzung in Gestalt des Entwicklungs- und Pflegeplans Tempelhofer Feld unter der Leitung der Senatsverwaltung für Stadtentwicklung und Grün Berlin, aber insbesondere auf die Grundlage der Diskussion von Zivilgesellschaft in Gestalt des 
Feldforums und der konstituierten Feldkoordination skizzieren und die für deren Umsetzung notwendigen Projekte begleiten soll (Senatsverwaltung für Stadtentwicklung und Umwelt 2016).

\subsection{Konfliktive Orte: zwischen der Vermeidung von Obdachlosigkeit und dem Recht auf Stadt}

Zwischen Sommer 2015 und Frühjahr 2016 nahmen die Ankunftszahlen von Geflüchteten in Berlin, aber auch in anderen deutschen Großstädten rasant zu. Die kurzfristige Bereitstellung von Grundversorgung für Tausende Menschen stellte die Berliner Verwaltung vor eine große verwaltungstechnische, logistische und humanitäre Herausforderung. Das sich vergrößernde Defizit an baulich geschlossenen, leer stehenden Immobilien für die Unterbringung von Geflüchteten zwang einzelne Bezirke dazu, bei der Neueröffnung von Notunterkünften auf größere Gebäude, Messehallen oder geschlossene Konversionsflächen auszuweichen. Im Falle der Notunterkunft Tempelhof - als der größten Flüchtlingsunterkunft in räumlicher und sozialer Nähe zum Tempelhofer Feld - kam es seit der Eröffnung Ende Oktober 2015 zu einer sukzessiven räumlichen Erweiterung der Unterbringungskapazitäten. Ende November 2015 waren in drei Hangars ca. 2.000 Menschen untergebracht. In der Debatte über die Jahreswende 2015/2016 sprach man von etwa 7.000 Bewohnern, die aufgrund fehlender Alternativen an Folge- oder Gemeinschaftseinrichtungen oder eines eklatanten Defizits an Wohnraum vorerst dauerhaft in Tempelhof unterkommen sollten (Der Tagesspiegel, 25.02.2016).

Mit den stagnierenden Zuwanderungszahlen von Geflüchteten ab dem zweiten Quartal 2016 kam es im Land Berlin zu einer merklichen Reduzierung der Ausbaudynamik von geplanten Notunterkünften und Aufnahmeeinrichtungen. Gleichzeitig wurde in der zweiten Jahreshälfte 2016 mit dem Aus-/Neubau von temporären und dauerhaften Gemeinschaftsunterkünften begonnen. Die politische Absicht, für die Unterkunft von Geflüchteten vorgesehene Turn- und Sporthallen durch Umverteilungs- bzw. Zentralisierungsvorgänge von der Belegung zu befreien, wurde Schritt für Schritt in die Tat umgesetzt. Im politischen Diskurs der Berliner Großen Koalition aus SPD und CDU wurde die Unterbringung der Geflüchteten Ende 2015 zur ersten, aber sehr zentralen Gretchenfrage des „Wie sollen wir das eigentlich schaffen?". Unter der Maxime der Vermeidung von Obdachlosigkeit und vor dem Hintergrund der vermeintlichen Alternativlosigkeit der Unterkunft wagte sich die amtierende Landesregierung an den gerade einmal anderthalb Jahre alten Gesetzesentwurf der Freihaltung des Tempelhofer Feldes heran.

In einer Gesetzesnovelle im Januar 2016 wurde schließlich die Bebauung des asphaltierten Vorfeldes des Flughafens zur Errichtung von temporären Gemein- 
schaftsunterkünften (sogenannte Tempohomes) gestattet. Die Bebauung des Feldes bzw. die Errichtung der temporären Infrastruktur auf der Fläche des Tempelhofer Feldes wird in der Novelle bis Ende 2019 legitimiert. Im Vorfeld des Beschlusses, aber insbesondere auch im Anschluss an den Gesetzesentwurf entfachte sich ein heftiger Streit zwischen der politischen Front und den zivilgesellschaftlichen Vertretungen (100\% Tempelhofer Feld). Nicht unbedeutend ist dabei, dass seit der Gesetzesnovelle im Januar 2016 ein Regierungswechsel von der Großen Koalition (SPD/CDU) hin zu R2G (SPD/Die Linke/Grüne) erfolgte, was einige der ursprünglichen Kritiker der Gesetzesnovelle in ihrer Rolle als Opposition mittlerweile in die Rolle der Gesetzesumsetzer gerückt hat. Ursprünglicher Plan war es, bis Juli 2017 auf dem Tempelhofer Feld 976 temporäre Wohncontainer für bis zu 1.100 Personen entstehen zu lassen. In die Container sollten hauptsächlich Bewohner der Hangars und der Notunterkunft im Internationalen Congress Centrum (ICC) umgesiedelt werden (vgl. Frühauf 2017).

Inmitten der Aushandlung um den Erhalt der Freifläche, die Verhinderung der Bebauung und das Recht auf Stadt lassen sich seit Mitte 2016 damit zunehmend Diskurselemente der Solidarisierung mit Geflüchteten und der offenen Kritik des Konzepts der Massenunterkünfte und Ghettoisierung von Geflüchteten beobachten. So kam es nicht nur zu einer Ausweitung der Interessenlage, sondern insbesondere auch zu einer Erweiterung an den Diskurspraktiken beteiligter Akteur*innen. Bei der Aushandlung des Ortes traten neben der bis dahin diskursdominierenden Initiativen 100\% Tempelhofer Feld insbesondere auch zivilgesellschaftliche Organisationen der ehrenamtlichen Flüchtlingshilfe oder gemeinnützige Sozialträger in den Vordergrund. Durch ihre z. T. variierenden Arbeits-, Begleit- und Unterstützungspraktiken und durch deren direkte Verflechtung mit der Zielgruppe der vor Ort wohnenden, neuen Nachbar*innen des Tempelhofer Feldes zeichneten sich letztere insbesondere durch den starken Raumbezug aus. Die Frage der Bereitstellung und Verhinderung von temporärem Wohnraum in Gestalt von Containern (sogenannte Tempohomes) wurde demnach sukzessiv auf die des Gestaltens einer neuen Nachbarschaft für neue Nachbar*innen der Geflüchtetenunterkunft ausgeweitet.

\subsection{Orte der interkulturellen Begegnung: Willkommenskultur im öffentlichen Raum}

Seit Sommer 2016 widmet sich ein durch die Senatsverwaltung beauftragtes Sozialunternehmen ${ }^{1}$ dem organisatorischen und inhaltlichen Aufbau einer Vernetzungs- und Kommunikationsstruktur, die eine Grundlage für die kommunikative

1 Das Projekt „Tempelhofer Feld - Aufbau einer Vernetzungs- und Kommunikationsstruktur“ 
und koordinative Abstimmung von niederschwelligen interkulturellen Begegnungsaktivitäten zwischen Neu- und Alt-Berliner*innen im Aktionsraum Tempelhofer Feld bietet. Grundlage des Projekts ist eine Entscheidung des Abgeordnetenhauses Berlin, die politische Maxime der Willkommenskultur mit integrationspolitischen Implikationen im öffentlichen Stadtraum Berlin umzusetzen. Die Entscheidung mündete in eine Ausschreibung und Suche nach einer Projektkonzeption, das sich der Einrichtung von Not- und Gemeinschaftsunterkünften im Aktionsraum des Tempelhofer Feldes und der damit verbundenen, qualitativ erweiterten Nutzung der öffentlichen Freifläche durch die „Gruppe“ der Geflüchteten widmen würde. Zur Begleitung, Mediation, Information, aber auch zur Kontrolle und Handhabung neuer Nutzer*innen und damit verbundener Praktiken wurde auf dem Tempelhofer Feld die Grundlage für ein lokales Integrationsmanagement gelegt. Ziel des Managens sollte die Aktivierung und Einbindung der breiten Gemengelage neuer und alter Initiativen, Vereine und Privaten mit Ortsbezug zum Feld in einer gemeinsamen Begegnungsarbeit innerhalb der Klammer „Willkommen auf dem Tempelhofer Feld" sein.

Das Projekt „Tempelhofer Feld - Aufbau einer Vernetzungs- und Kommunikationsstruktur" gliedert sich in die drei Elemente des lokalen Integrationsmanagements: Sprachmittlung, mobile Beratung und Vernetzung. Nach inhaltlicher Sichtung und Analyse der veröffentlichten Projektberichte der implementierten Vernetzungs- und Kommunikationsmodule lassen sich folgende qualitativen Ergebnisse herausstellen (vgl. Grün Berlin 2016):

- Sprachmittlung: Die Sprachmittlungs- und Netzwerkarbeit durch ein mehrsprachiges Projektteam bildete den zentralen Baustein für die Einbindung der Geflüchteten in verschiedene Begegnungsformate. Auch wenn sich nur ein Teil der aufgesuchten Gruppen in den regelmäßigen Aktivitäten zeigte - was oftmals mit Schulzeiten, Amtsterminen etc. in Verbindung stand -, ließe sich die Aktivierung und Begleitung von Einzelnen oder ganzen Klein- und Großgruppen zu Veranstaltungen oder zum Zugangsort des Info-Pavillons als communitybildender Brückenschlag verzeichnen. Der Kontakt- und Vertrauensaufbau war bei der Aktivierung aller Nutzer*innengruppen in öffentlich zugänglichen Begegnungsformaten von entscheidender Bedeutung.

wird im Auftrag der Grün Berlin GmbH durch das Sozialunternehmen interkular gGmbH ausgeführt. Der Autor steht dem Sozialunternehmen in geschäftsführender Rolle vor. Der Autor verfolgt die Geschehnisse rund um das Tempelhofer Feld seit 2015 nicht nur wissenschaftlich, sondern auch durch die Projektumsetzung und konnte empirische Daten aus unzähligen Gesprächen mit dem diskutierten Akteursfeld in die Erkenntnisse des Beitrags einfließen lassen. 
- Mobile Beratung: Der Baustein der mobilen Beratung bildete die inhaltliche Basis der Projektarbeit und stellte für viele Nutzer*innengruppen das personelle Gesicht der Begegnungsarbeit dar. Durch die Erweiterung der Praktiken der Informations- und Wissensvermittlung um ein bedarfsorientiertes Angebot von Sport- und Spielaktivitäten sei der Ort des Info-Pavillons zunehmend zum zentralen Ort der Begegnung und des Austauschs geworden.

- Vernetzung: Vor dem Hintergrund der projektspezifischen Rahmenbedingungen hat sich die Netzwerkarbeit mit Initiativen und Vereinen der umliegenden Nachbarschaften unterschiedlich intensiv gestaltet. Die Herausforderungen des Netzwerkaufbaus und der Netzwerkpflege reichten von schwer überwindbaren Vorbehalten bis hin zu großer Kooperationsbereitschaft gegenüber dem Projektziel. Mittels der Durchführung eines Runden Tisches konnte ein produktiver Schulterschluss zwischen unterschiedlichen Akteursgruppen hergestellt werden. Ebenso konnten erste lösungsorientierte Ansätze für die Umsetzung kombinierter interkultureller Begegnungsformate eines ergebnisoffenen lokalen Integrationsmanagements geschaffen werden. Schließlich habe der mehrsprachige Veranstaltungskalender dabei geholfen, ein ansprechendes Format für die Kommunikation von Begegnungsformaten zu schaffen. Die Reichweite des Veranstaltungskalenders kann nur schwer eingeschätzt werden. Allerdings sei seine positive Wirkung in Unterkünften deutlich geworden. Gleichzeitig stehen Reichweite und Akzeptanz des Veranstaltungskalenders in Verbindung mit der Vielfalt der Begegnungsformate.

Aufbauend auf den Projekterfahrungen der ersten Saison der Willkommenskultur auf dem Tempelhofer Feld wurden im abschließenden Projektbericht 2016 konkrete Handlungsempfehlungen für die Einrichtung und die qualifizierte Weiterentwicklung der Vernetzungsarbeit formuliert (vgl. Grün Berlin 2016). Konkretes Ergebnis dieser Handlungsempfehlungen ist es, das Tempelhofer Feld als Ort der interkulturellen Begegnung zu qualifizieren. Aufbauend auf den konsolidierten Projekten auf dem Tempelhofer Feld und den im Sommer 2016 angeschobenen Begleitungsformaten werden Veranstaltungen interkultureller Begleitungsaktivitäten an zentralen Orten der Freifläche empfohlen. Dazu zählen etwa Dialogveranstaltungen zu Ehrenamt oder bürgerschaftlichem Engagement, interkulturelle Bildungsspaziergänge oder Aktionstage mit Musik, Kinderprogramm und Erfahrungsaustausch zwischen lokalen Akteur*innen. An diesem neuen Ort der Begegnung kommt im Falle des Tempelhofer Feldes dem Info-Pavillon eine zentrale Rolle zu. Der Info-Pavillon war von der landeseigenen Betreibergesellschaft Grün Berlin GmbH ursprünglich als reiner Informationsort vorgesehen gewesen. Durch die seit 2016 umgesetzten Praktiken der lokalräumlichen Interaktion und Teilhabe 
- wie das Verleihen von Spiel- und Sportgeräten oder das Durchführen von Veranstaltungen und regelmäßigen Aktivitäten - ist der Info-Pavillon nicht nur funktional aufgewertet, sondern zu einem Zugangsort und Treffpunkt interkultureller Begegnung geworden. Die Teilhabe und Aktivierung neuer Nutzer*innengruppen im öffentlichen Freiraum kann daher als Chance für die Gestaltung neuer Nachbarschaften gesehen werden. Insbesondere die Einbindung der Gruppe der Bewohner*innen der Tempohomes schafft Gelegenheit, das Tempelhofer Feld vom Sommer 2018 an noch weiter als Ort der interkulturellen Begegnungsarbeit zu qualifizieren. Dabei ist die Berücksichtigung der Bedarfe von Geflüchteten von großer projektspezifischer Bedeutung. Durch die Schaffung und Umsetzung von kulinarischen und interkulturellen Begegnungsformaten - wie Teestunden, Picknick, gemeinsamem Musizieren - konnten Anreize für die Teilhabe am Entstehungsprozess der neuen Nachbarschaft geschaffen werden. Sie bilden wichtige Erfahrungswerte für ein lokales Integrationsmanagement im direkten Sozialraum von Geflüchteten-Unterkünften und lassen sich mitunter auf andere beteiligungsorientierte Projekte des Nachbarschaften-Machens in der Co-Produktion von öffentlichen und zivilgesellschaftlichen Akteur*innen übertragen (z. B. „BENN - Berlin Entwickelt Neue Nachbarschaften").

\subsection{Neue Nachbarschaften praktikentheoretisch denken}

„Integration findet vor Ort statt“ (Bommes 2008: 160) - und vor Ort sind Migrant*innen nicht nur in Arenen urbaner Ökonomie, Bildungs- und Arbeitssysteme sowie soziale Netzwerke eingebunden, denen die wissenschaftliche Debatte integrationsfördernde Effekt beimisst (vgl. Gesemann 2016). Das Vor-Ort ist vor allem die lokale Ebene, auf der sozialer Austausch, interkulturelle Begegnung sowie gesellschaftliche Teilhabe stattfindet, während zeitgleich gesellschaftlicher Konflikt diskursiv und alltagspraktisch verhandelt wird. Das Lokale ist daher auch nicht grundlos der Ort, an dem die politische Maxime „Wir schaffen das“ pragmatische Lösungen für die Integrationsaufgabe sucht. Im empirischen Rückblick auf die beiden Fallbeispiele - die diskursive Aushandlung der Unterbringung von Geflüchteten in der Notunterkunft des ehemaligen Flughafens Tempelhof einerseits, und Gestaltung der interkulturellen Begegnung auf dem Tempelhofer Feld andererseits - treten das Machen und Werden neuer Orte der Integration in den Mittelpunkt der Betrachtung. Mithilfe der praktikentheoretischen Perspektive auf diesen produktiven Prozess der Entstehung neuer Orte des Lokalen wird der Blick auf alltägliche Diskurse, Materialitäten und soziale Akteur*innen freigelegt. Sie sind jeweils verbunden mit einem bestimmten Tun und Sagen. Dazu zählen Sprechakte des Protests gegen die Bebauung öffentlicher Freiflächen, das Niederschreiben und Einführen von Gesetzesnovellen zur Vermeidung von Obdachlosigkeit oder 
auch niederschwellige Begegnungsaktivitäten beim Fußballspielen, Musizieren oder gemeinsamen Kochen. Die Theorie der sozialen Praktiken sieht das Entstehen sozialer Räume als konstitutiven Prozess der Verknüpfung von eben diesen Praktiken und Ordnungen. Im Beispiel des Tempelhofer Feldes lässt sich empirisch schließlich zeigen, wie neue soziale Räume mit bestimmten integrativen Schlüsselrollen - in diesem Fall Nachbarschaften - in einzelnen diskursiven und aktivitäts- und veranstaltungsbezogenen Praktiken-Ordnungen-Bündeln der Begegnung, des Austauschs und der Teilhabe entstehen.

\section{Beobachtungen zusammenbringen}

Das komplexe Föderalsystem in Deutschland rahmt die Governance der Integration maßgeblich. So stehen vertikale Kompetenzverteilungen zwischen Bund, Ländern und Kommunen den horizontalen Kompetenzverteilungen zwischen den Ressorts der Integration z. T. entgegen. Gleichzeitig ist es auch eben diese Struktur, die Deutschland zu einem lebendigen Laboratorium für Innovationen im Integrationsbereich macht. Anders als in anderen Ländern der EU gibt es zahlreiche sich z. T. ergänzende Integrationsparadigmen. Insbesondere in der Erweiterung der integrationspolitischen Frage um die aktuelle Flüchtlingspolitik, die zwischen 2015 und 2016 im Krisenmodus betrieben wurde, liegt eine Chance für eine Erneuerung und Weiterentwicklung der Integrationspolitik. Die offene Struktur der derzeitigen kommunalen Integrationspolitik schafft Möglichkeitsräume für Innovationen.

Mit Blick auf die Schüsselrolle der lokalen Ebene der Nachbarschaft diskutiert der Beitrag im Kontext des Tempelhofer Feldes zwei Beispiele der Entstehung neuer Nachbarschaften. Aus der Perspektive der kommunalen Integrationspolitik wurde dabei der Frage nachgegangen, wie sich die gesellschaftspolitische Maxime der Willkommenskultur in das diskursive und anwendungsorientierte Feld der Begegnung und Teilhabe einwebt. In der praktikentheoretischen Lesart der Fallbeispiele spannen die planerische Setzung einer temporären Gemeinschaftsunterkunft und der Auftrag zum Aufbau einer Vernetzungs- und Kommunikationsstruktur einen Möglichkeitsraum für die Gestaltung der niederschwelligen Begegnungsarbeit auf. Dabei gelangen durch die diskursive Verhandlung und die erfahrungsbasierte Verschriftlichung interkultureller Ansätze der Begegnungsarbeit akteurs- und ressourcenbezogene sowie an die pluralen Lebenswirklichkeiten angelehnte Sichtweisen auf das Nachbarschaften-Machen in die planungspraktische Realität. Mit dieser Erweiterung lässt sich auch eine planungspraktische Verschiebung hin zu einem kultursensiblen Verständnis von bespielbarem Sozialraum anstelle von strukturellem Planungsraum vermuten. 
Der Krisenmodus der Versorgung, Unterbringung und Einbindung von Geflüchteten ist daher mitunter auch als Möglichkeitsrahmen zu lesen. Denn anstatt den Krisenmodus weiterzufahren oder auch „nur“ diskursiv zu verteufeln, kann ein wissenschaftlich gestützter Erfahrungsaustausch über Praktiken der Begegnung und Teilhabe entlang der Schnittstellen von Verwaltung und Zivilgesellschaft eine stärker pragmatische Debatte um neue, integrationspolitische Wege auf der Nachbarschaftsebene anstoßen. Damit bietet die „Flüchtlingskrise“ der Jahre 2015 und 2016 ein Kontinuum zwischen Kritik und Vermittlermodus. Der Modus bietet viele Ansatzpunkte dafür, wie aktuelle und zukünftige Herausforderungen der Integration durch eine sozialraumorientierte Teilhabe und Mitgestaltung bewältigt werden können. Neben der politischen Maxime einer Anerkennungs- und Willkommenskultur und einer auf Vielfalt ausgerichteten Integrationspolitik treten plurale Akteur*innen- und Praktikengeflechte des Nachbarschaften-Machens in den Vordergrund.

Schließlich macht sich der Beitrag auf die Suche nach einer spezifischen Übersetzungs- und Kommunikationsmethodik, welche eine Planungspraxis des kommunalen Integrationsmanagements um den wissenschaftlich gestützten Erfahrungsaustausch erweitert. Um der Querschnittsaufgabe der Integration gerecht zu werden, stellt diese Methodik das Verwoben-Sein komplexer Aktivitäten (Praktiken) mit der großen Gemengelage aus Akteur*innen unterschiedlicher Verwaltungsebenen, gesetzlichen Rahmenbindungen und diskursiven Formationen (Arrangements bzw. Ordnungen) in den Mittelpunkt der Betrachtung des gesellschaftsrelevanten Phänomens der Integration von Zugewanderten. Auf der Suche nach dieser Übersetzungsmethodik des lokalen Integrationsmanagements spricht sich der Beitrag für das Potenzial der methodologischen Erweiterung des städtischen Governance-Ansatzes um praktikentheoretische Theoriebausteine aus. Kommunale Integrationspolitik lässt sich entsprechend als plurale Verflechtung von neuen Akteur*innenkonstellationen und Praktiken lesen. In diesem Geflecht entstehen sites der Begegnung und Teilhabe. Im Zeichen eines relationalen Raumund Handlungsbezugs bilden die sites ganz praxisnahe Laboratorien des Nachbarschaften-Machens und erweitern das Spektrum der Governance-Arenen der Integration um Erprobungsräume der Co-Produktion einer pluralen Stadtgesellschaft. In diesen Arenen werden Herausforderungen, Bedarfe und Lösungen nicht etwa getrennt voneinander betrachtet, sondern treten in den Mittelpunkt der Betrachtung des Sozialräumlichen. Auf der Suche nach dem „So schaffen wir das“ ist die Pluralität der Akteur*innen- und Handlungszusammenhänge in konkreten Praxiszusammenhängen des lokalen Integrationsmanagements schließlich als innovatives Feld des Entstehens neuer Nachbarschaften zu skizzieren. 


\section{Literatur}

Aumüller, J. \& Gesemann, F. (2014): Integrationspotentiale ländlicher Regionen im Strukturwandel. Darmstadt.

Bommes, M. (2008): „Integration findet vor Ort statt“ - über die Neugestaltung kommunaler Integrationspolitik. In: Bommes M. et al. (Hrsg.): Migrationsreport 2008. Fakten - Analysen - Perspektiven. Frankfurt am Main: 159-194.

Buckow, W. D., Heck, G., Schulze, E. \& Yildiz, E. (Hrsg.): Neue Vielfalt in der urbanen Stadtgesellschaft. Wiesbaden.

Deffner, V. (2013): Quartiere als soziale Räume. Sozialgeographische Reflexion. In: Deffner, V. \& Meisel, U. (Hrsg.). StadtQuartiere: Sozialwissenschaftliche, ökonomische und städtebaulich-architektonische Perspektiven. Essen: 83-98.

De Mazière, T. (2015): Pressekonferenz von Bundesminister am 19.08.2015. https://goo.gl/ zF1CUo. Letzter Zugriff: 24.8.2017.

Der Tagesspiegel (25.2.2016): Die nächsten beiden Hangars werden bald belegt. Abrufbar unter: https://goo.gl/aSvZnv. Letzter Zugriff 24.08.2017

Everts, J., Lahr-Kurten, M., \& Watson, M. (2011): Practice matters! Geographical inquiry and theories of practice. Erdkunde, 65(4): 232-334.

Frühauf, H. (2017): Tempohomes, eine Glaubensfrage (26.4.2017). Abrufbar unter: https://goo.gl/FySJwP. Letzter Zugriff: 24.8.2017.

Gabriel, S. (2015): „VIDEO-PODCAST \#3 // FLÜCHTLINGSPOLITIK“. Abrufbar unter: https://sigmar-gabriel.de/video-podcast-3-fluechtlingspolitik/. Letzter Zugriff: 24.8.2017.

Gesemann, F. (2016): Kommunale Integrationspolitik. In: Brinkmann, H. U. \& Sauer, M. (Hrsg.): Einwanderungsgesellschaft Deutschland. Entwicklung und Stand der Integration. Wiesbaden: 281-309.

Grün Berlin (2016): Abschlussbericht im Projekt „Tempelhofer Feld - Aufbau einer Kommunikations- und Vernetzungsstruktur“. 30.11.2016, Berlin. Abrufbar unter: https://goo.gl/j9dyTE. Letzter Zugriff: 24.8.2017.

Heißler, J. (2016): Merkels drei große kleine Worte. In: tagesschau.de (31.8.2016). Abrufbar unter: https://goo.gl/FSyNyK. Letzter Zugriff: 24.8.2017.

Maus, G. (2015): Erinnerungslandschaften: Praktiken ortsbezogenen Erinnerns am Beispiel des Kalten Krieges. In: Kieler Geographische Schriften, Band 127. Kiel.

Merkel, A. (2015): Sommerpressekonferenz von Bundeskanzlerin Merkel. https://goo.gl/Sj FiCN. Letzter Zugriff: 31.10.2017.

Saunders, D. (2010): Arrival city. How the largest migration in history is reshaping our world. London.

Schatzki, T. (2002): The Site of the Social. A Philosophical Account of the Constitution of Social Life and Change. Pennsylvania.

Senatsverwaltung für Justiz und Verbraucherschutz (2014): Gesetz- und Verordnungsblatt für Berlin: Gesetz zum Erhalt des Tempelhofer Feldes (ThF-Gesetz). Berlin.

Senatsverwaltung für Stadtentwicklung und Umwelt (2016): Tempelhofer Feld: Entwicklungs- und Pflegeplan. Berlin.

Schiffauer, W., Eilert, A. \& Rudloff, M. (Hrsg.): So schaffen wir das - eine Zivilgesellschaft im Aufbruch. 90 wegweisende Projekte mit Geflüchteten. Bielefeld. 
Schnur, O. (2008): Quartiersforschung im Überblick: Konzepte, Definitionen und aktuelle Perspektiven. In: Schnur, O. (Hrsg.): Quartiersforschung. Zwischen Theorie und Praxis. Wiesbaden: 19-51.

Schnur, O. (2012): Quartier und Nachbarschaft. In: Eckhart, F. (Hrsg.): Handbuch Stadtsoziologie. Wiesbaden: 449-474.

Vogelpohl, A. (2008): Stadt der Quartiere? Das Place-Konzept und die Idee von urbanen Dörfern. In: Schnur, O. (Hrsg.): Quartiersforschung. Zwischen Theorie und Praxis. Wiesbaden: 69-86.

Open Access Dieses Kapitel wird unter der Creative Commons Namensnennung 4.0 International Lizenz (http://creativecommons.org/licenses/by/4.0/deed.de) veröffentlicht, welche die Nutzung, Vervielfältigung, Bearbeitung, Verbreitung und Wiedergabe in jeglichem Medium und Format erlaubt, sofern Sie den/die ursprünglichen Autor(en) und die Quelle ordnungsgemäß nennen, einen Link zur Creative Commons Lizenz beifügen und angeben, ob Änderungen vorgenommen wurden.

Die in diesem Kapitel enthaltenen Bilder und sonstiges Drittmaterial unterliegen ebenfalls der genannten Creative Commons Lizenz, sofern sich aus der Abbildungslegende nichts anderes ergibt. Sofern das betreffende Material nicht unter der genannten Creative Commons Lizenz steht und die betreffende Handlung nicht nach gesetzlichen Vorschriften erlaubt ist, ist für die oben aufgeführten Weiterverwendungen des Materials die Einwilligung des jeweiligen Rechteinhabers einzuholen.

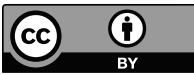

